\title{
Caractérisation phénotypique de 52 souches des Bacillus isolées à partir de racines fraîches de manioc cultivées en Côte d'Ivoire
}

\author{
Anne Marie Koua $\mathrm{ABE}^{1}$, Justine Bomo ASSANVO ${ }^{1 *}$, Moussa $\mathrm{SANOGO}^{2}$ et \\ Kouamé Mathias KOFFI ${ }^{1}$ \\ ${ }^{l}$ Laboratoire de Biochimie et Sciences des Aliments, UFR Biosciences, \\ Université Félix Houphouët-Boigny, 22 BP 582 Abidjan 22, Côte d'Ivoire. \\ ${ }^{2}$ Laboratoire Central Vétérinaire de Bingerville, Laboratoire National d'Appui au Développement Agricole \\ (LANADA), BP 206 Bingerville, Côte d'Ivoire. \\ *Auteur correspondant, E-mail: justinebomo2015@gmail.com, Tél: (+225) 07786755.
}

\section{RESUME}

Dans la plupart des pays africains, le manioc constitue une source d'énergie accessible et beaucoup consommé frais ou sous forme de mets confectionnés. L'objectif de cette étude est de contribuer à l'amélioration de la qualité sanitaire des aliments de grande consommation comme le manioc en Côte d'Ivoire. Ainsi, 52 souches de Bacillus isolés à partir d'échantillons de manioc frais ont été caractérisées à l'aide des techniques combinées d'identification morphologiques et biochimiques. Sur la base des critères morphologiques et biochimiques, les souches suspectées ont été réparties en espèces de Bacillus cereus (55,8\%), Bacillus brevis (17,3\%), Bacillus subtilis (13,5\%), Bacillus coagulans $(9,6 \%)$ et Bacillus spp $(3,8 \%)$. La prédominance de Bacillus cereus interpelle, car cette espèce est souvent impliquée dans les cas de toxiinfections alimentaires. Toutefois, les espèces comme Bacillus coagulans, Bacillus subtilis et Bacillus brevis offrent des prestations assez intéressantes: Bacillus subtilis représente un moyen de lutte biologique contre les maladies fongiques des plantes et est utilisé comme starter dans la production de plusieurs aliments condiments africains, Bacillus coagulans est un véritable probiotique, Bacillus brevis produit un antibiotique, la thyrothricine qui soulage les maux de gorge.

(C) 2018 International Formulae Group. All rights reserved.

Mots clés: Identification, racines de manioc, Bacillus spp, toxi-Infection alimentaires.

\section{Phenotypic characterization of 52 strains of Bacillus isolated from fresh cassava roots grown in Côte d'Ivoire}

\begin{abstract}
In most of Africa countries, cassava is an accessible source of proteins which is consumed fresh or cooked. The aim of the current study is to contribute to the improvement the quality and safe consumption of cassava in Côte d'Ivoire. Fifty-two strains of Bacillus isolated from cassava were characterized using the morphological and biochemical identification techniques. On the basis of morphological and biochemical criteria, the suspected strains were classified as Bacillus cereus (55.8\%), Bacillus brevis (17.3\%), Bacillus subtilis $(13.5 \%)$, Bacillus coagulans $(9.6 \%)$ and Bacillus sp (3.8\%). This high presence of Bacillus cereus is a
\end{abstract}


problem because this species of Bacillus is often involved in cases of foodborne diseases. However, species such as Bacillus coagulans, Bacillus subtilis and Bacillus brevis have interesting features: Bacillus subtilis is a means of biological control of fungal diseases of plants and is used as a starter in the production of several African condiment foods, Bacillus coagulans is a true probiotic, Bacillus brevis produces an antibiotic, thyrothricin which relieves the ailments of throat.

(C) 2018 International Formulae Group. All rights reserved.

Keywords: Identification, cassava roots, Bacillus spp, alimentary toxi-Infections.

\section{INTRODUCTION}

Originaire d'Amérique du Sud, le manioc (Manihot esculenta, Crantz) est une plante tropicale appartenant à la famille des Euphorbiacées avec plus de 300 variétés connues. Selon la FAO (2016), la production annuelle mondiale est estimée à près de 228 138068 tonnes/an dont 2,4 millions de tonnes/an en Côte d'Ivoire. Dans ce pays, le manioc occupe le $2^{\text {ème }}$ rang au niveau des cultures vivrières après l'igname (FAO, 2016).

Les racines de manioc sont beaucoup consommées dans les pays africains (Agre et al., 2015) car elles constituent des sources d'énergie peu coûteuses. Elles sont souvent consommées fraîches comme à l'Ouest de la Côte d'Ivoire ou sous forme de mets confectionnés à partir des racines (Burns et al., 2012), dont les plus connus sont le kokondé, le gari, l'attiéké, l'attoukpou, le placali et le foutou (Assanvo et al., 2006). Les racines de manioc doux sont souvent consommées de préférence comme un goûter entre les repas, au champ, juste après la récolte, ou en ville, vendues par des commerçantes ambulantes comme légumes. Dans la plupart des cas, elles sont consommées crues après épluchage. La consommation $\mathrm{du}$ manioc sous forme de racines «fraîches» (cru, cuit ou rôti) est estimée à environ 30\% (Pistretto, 2007).

Dans le manioc, on peut trouver des souches de bactéries dont Bacillus. Celles-ci constituent des hôtes normaux du sol dans lequel elles peuvent persister très longtemps grâce à leurs spores et leur état saprophyte très commun de l'air. Sur le manioc, les Bacillus disposent d'un substrat nutritif et jouent un rôle important dans la dégradation tissulaire du manioc (Obilie et al., 2003). Cette dégradation aboutit à la libération de composés cyanogènes grâce aux enzymes telles que les polygalacturonases, les pectines estérases, les cellulases et les amylases (Obilie et al., 2003). Les Bacillus contribuent ainsi à la détoxication du manioc en permettant un contact facile entre les linamarases et les glucosides cyanogéniques. Par leur action, les souches de Bacillus ont également la capacité de produire des molécules d'intérêt biotechnologique, ce qui constituent l'une des propriétés les plus prisée de ces souches (Waites et al., 2001). Les molécules produites peuvent être utilisées dans le domaine de l'agriculture notamment dans la lutte biologique (Aboussaid et al., 2009) mais aussi dans le domaine agro-alimentaire et médical. La plupart des souches de Bacillus sont inoffensives ou bénéfiques pour l'organisme. Par exemple, Bacillus subtilis est utilisé comme starter dans la production de plusieurs aliments condiments africains (Amoa-awua et al., 2006; Azokpota et al., 2006; 2008; Parkouda et al., 2009). Il existe cependant certaines espèces pathogènes comme Bacillus anthracis et Bacillus cereus qui peuvent être à l'origine de diverses maladies comme la maladie du Charbon bactéridien et les ToxiInfections Alimentaires (Guinebretière et al., 2010; Hafidi et al., 2013).

Les Bacillus ont fait l'objet de nombreux travaux scientifiques sous divers aspects (Stein, 2005; Azokpota et al., 2007; Oyeleke et al., 2011;Vinoj et al., 2013; Yobouet et al., 2016). En Côte d'Ivoire, les études effectuées sur les Bacillus du manioc ont été réalisées en général sur le manioc fermenté et certains de ses produits dérivés comme l'attiéké (Assanvo et al., 2006; Coulin 
et al., 2006; Kouamé et al., 2012; Kacou et al., 2017). Peu de travaux scientifiques se sont intéressés aux souches du manioc frais et à leur impact sur la santé des consommateurs.

Cette étude se propose de contribuer à l'amélioration de la qualité sanitaire des aliments de grande consommation comme le manioc en Côte d'Ivoire. Dans cette optique, les caractéristiques morphologiques et biochimiques des souches de Bacillus isolées à partir de racines fraîches de manioc ont été identifiées en vue d'une évaluation de leur impact sur la santé des populations consommatrices.

\section{MATERIEL ET METHODES Souches étudiées}

Un total de 52 souches de Bacillus préalablement isolées à partir de racines fraîches de manioc et conservées à $-80{ }^{\circ} \mathrm{C}$ dans du glycérol dans une banque de souches bactériennes, ont servi dans le cadre de cette étude.

Ces souches ont été chauffées dans du bouillon viande-foie à $80{ }^{\circ} \mathrm{C}$ pendant 15 minutes pour obtenir des souches pures aptes à toute sorte de test d'identification. Après cette purification et revivification des souches, elles sont repiquées sur les géloses Trypticase de soja (TCS), gélose Mossel et gélose au sang par stries d'épuisement, puis incubées à l'étuve à $37^{\circ} \mathrm{C}$ pendant 24 heures, sauf celles repiquées sur le milieu Mossel qui ont été incubées à $30{ }^{\circ} \mathrm{C}$ pendant 48 heures. Une observation des colonies a été faite après le temps d'incubation.

\section{Caractérisation morphologique des souches \\ L'identification morphologique a} d'abord consisté en un examen macroscopique par l'observation directe à l'œil nu de l'aspect des colonies obtenues sur le milieu TCS, la gélose Mossel et la gélose au sang après incubation. La gélose Mossel a servi pour l'isolement de Bacillus cereus. La gélose au sang a été utilisée pour mettre en évidence le caractère de l'hémolyse des Bacillus. Ensuite, un examen microscopique des colonies à l'état frais et une coloration de Gram ont été réalisés.

\section{Caractérisation biochimique des souches}

Pour la caractérisation biochimique, plusieurs tests ont été réalisés. Il s'agissait des tests de la catalase, de l'oxydase et du type respiratoire.

Le test de la catalase a consisté à mettre en suspension une colonie de culture bactérienne dans une goutte d'eau oxygénée à $3 \%$. Un résultat positif visible à l'œil nu est un dégagement d'oxygène qui se traduira par une effervescence, l'absence d'effervescence est un résultat négatif.

Le test de l'oxydase a consisté à mettre sur une lame de verre bien nettoyée, un disque pré-impregné d'un réactif incolore (la $\mathrm{NN}$ diméthyl-paraphénylène diamine). Avec une pipette Pasteur, on a prélevé une colonie sur un milieu solide qui a été déposée doucement sur le disque. Une réaction positive se traduit par l'apparition d'une coloration rose violacé.

Le test du type respiratoire a consisté préalablement en une régénération du milieu viande foie (VF) par un chauffage durant 30 minutes au bain-marie bouillon. L'ensemencement a été réalisé à l'aide d'une pipette Pasteur plongée au fond du tube, puis remontée en décrivant une spire, de façon à ensemencer uniformément le milieu sur toute la hauteur. Après refroidissement, le milieu est mis en incubation à $37{ }^{\circ} \mathrm{C}$ pendant 24 à 48 heures.

Aussi, cette identification biochimique a été menée par une galerie classique, qui a regroupé les tests du glucose et du lactose, du citrate, du mannitol-mobilité-nitrate et du test de l'urée-indole.

Le test du glucose et du lactose a consisté à ensemencer une colonie dans le milieu Kliger-Hajna en réalisant une piqure centrale dans le culot et des stries serrées sur la pente. Les tubes ont été légèrement fermés. L'incubation a été réalisée à $37^{\circ} \mathrm{C}$ pendant 24 heures.

Le test du citrate a consisté à ensemencer une colonie bactérienne sur la pente du milieu par stries longitudinales. Les tubes sont légèrement fermés. L'incubation se fait à $37^{\circ} \mathrm{C}$ pendant 24 heures. Pour le test du mannitol-mobilité-nitrate, une colonie de la souche a été ensemencée par piqure centrale 
dans le tube de mannitol-mobilité-nitrate et incubée à $37{ }^{\circ} \mathrm{C}$ pendant 24 heures.

\section{Analyses statistiques}

L'ensemble des résultats a été compilé dans une base de données Excel (Office 2013). Les proportions relatives des Bacillus caractérisés ont été déterminées par une analyse statistique descriptive.

\section{RESULTATS}

\section{Caractéristiques souches étudiées \\ morphologiques des}

Sur le milieu TCS, les boîtes de Pétri ont révélé trois (3) types de colonies (Figure $1)$.

Au total, 24/52 souches ont présenté des colonies moyennes irrégulières à surface sèche, de couleur blanchâtre (Figure 1A), $14 / 52$ souches ont des colonies moyennes régulières blanchâtres bombées à surface gélatineuse (Figure $1 \mathrm{~B}$ ), 14/52 souches ont présenté des colonies moyennes rondes, planes, à contour circulaires, régulières, à surface sèche, de couleur blanchâtre (Figure $1 \mathrm{C)}$.

Sur le milieu Mossel, 29/52 souches sont apparues sous forme de moyennes colonies rosées irrégulières à surface sèche ou parfois crémeuse, 7 souches ont présenté de moyennes colonies jaunâtres irrégulières à surface sèche ou parfois crémeuse. Seize (16) des souches avaient des moyennes colonies incolores irrégulières à surface sèche ou parfois crémeuse (Figure 2).

Sur la gélose au sang, toutes les souches ont présenté une hémolyse positive (Figure 3).

Aussi, l'examen à l'état frais et de la coloration de Gram, ont permis de révéler que toutes les souches se présentaient sous forme de bâtonnets droits à extrémités arrondies, de taille variable à Gram positif, mobiles avec d'une part, des spores ovales centrales ou subterminales non déformantes et d'autre part, des spores terminales déformantes. Ces bacilles étaient soit regroupées en diplobacilles, soit regroupées en chaînettes, soit isolées.

L'ensemble des caractères phénotypiques comprenant la morphologie des colonies, la forme, le Gram et la mobilité des souches (Tableau 1), la production des spores révèle donc les traits caractéristiques des Bacillus. Les résultats sont conformes aux caractéristiques générales des souches du genre Bacillus appartenant au groupe I (groupe de Bacillus cereus) et du groupe II (groupe de Bacillus subtilis).

\section{Caractéristiques biochimiques des souches étudiées}

Les différents résultats des tests biochimiques (Tableau 1) ont montré que toutes les souches analysées sont positives au test de la catalase. Certaines ont enregistré une oxydase positive, tandis que d'autres étaient négatives. En ce qui concerne le test du type respiratoire, certains tubes ont présenté des cultures dans la zone superficielle, ce qui définit des souches aérobies stricts et d'autres tubes sur toute la hauteur du tube, définissant des souches aéroanaérobies facultatifs. Ces résultats sont conformes avec les caractéristiques des Bacillus.

Pour pousser l'identification, des tests biochimiques définissant une galerie classique ont été réalisés pour identifier les différentes souches de Bacillus. Ces tests d'identification des Bacillus selon Barrow et Feltham (1993) ont été confrontés avec les résultats obtenus dans les tests précédemment effectués et cela a permis de mettre en évidence la présence de Bacillus cereus, Bacillus brevis, Bacillus coagulans et Bacillus subtilis, présents dans le manioc.

Sur les 52 souches étudiées, on a enregistré une population prédominante de Bacillus cereus avec 29 souches soit 55,8\%, 9 souches de Bacillus brevis soit 17,3\%, 7 souches de Bacillus subtilis soit 13,5\%, 5 souches de Bacillus coagulans soit 9,6\% et 2 souches de Bacillus sp. soit 3,8\% (Figure 4). 

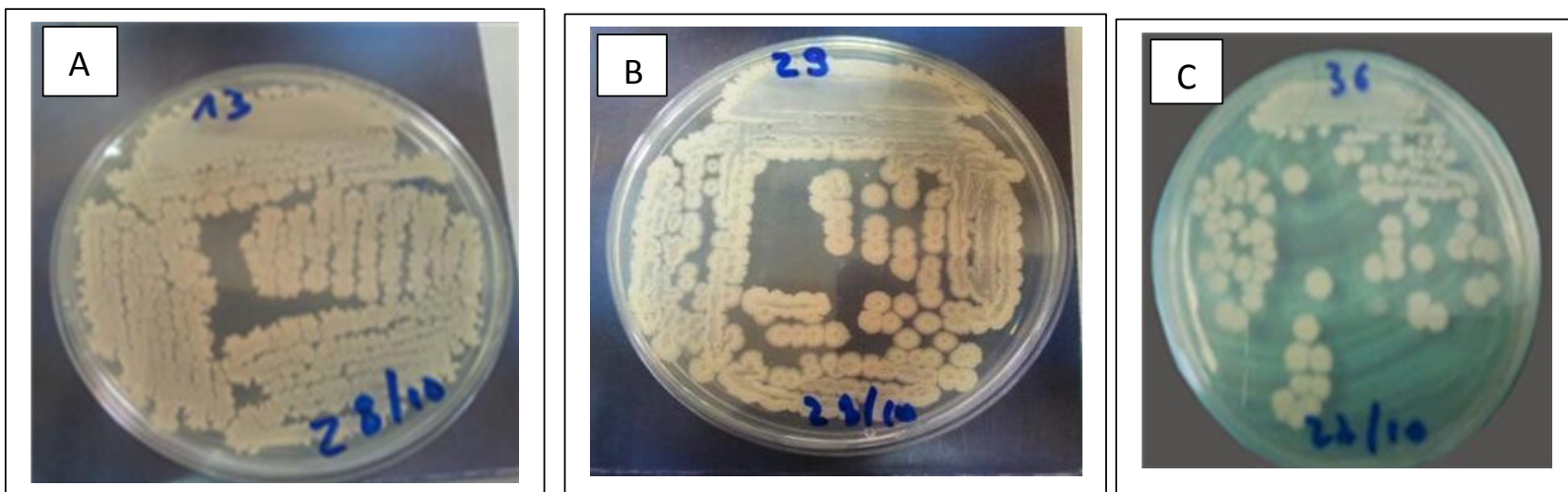

Figure 1: Photographies présentant l'aspect morphologique des colonies obtenues sur TCS après incubation de 24 h. A: colonie type1, B: colonie type 2, C:colonie type 3 (Cl. Abé, 2016).
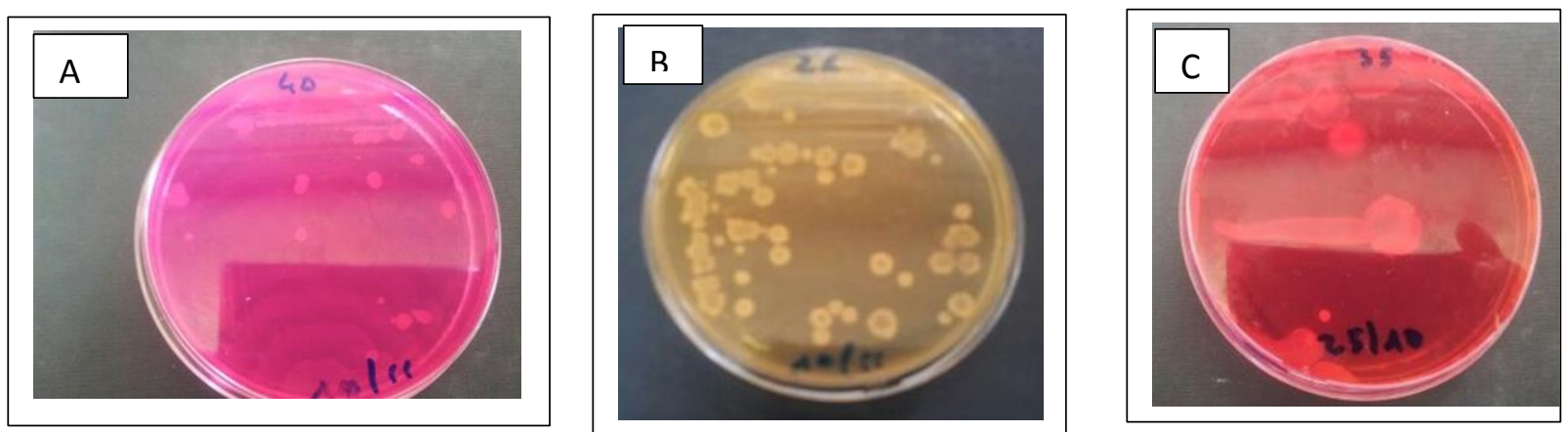

Figure 2: Photographies présentant l'aspect morphologique des colonies obtenues sur Mossel après incubation. A : colonie type 1, B : colonie type 2, C: colonie type 3 (Cl: Abé, 2016).
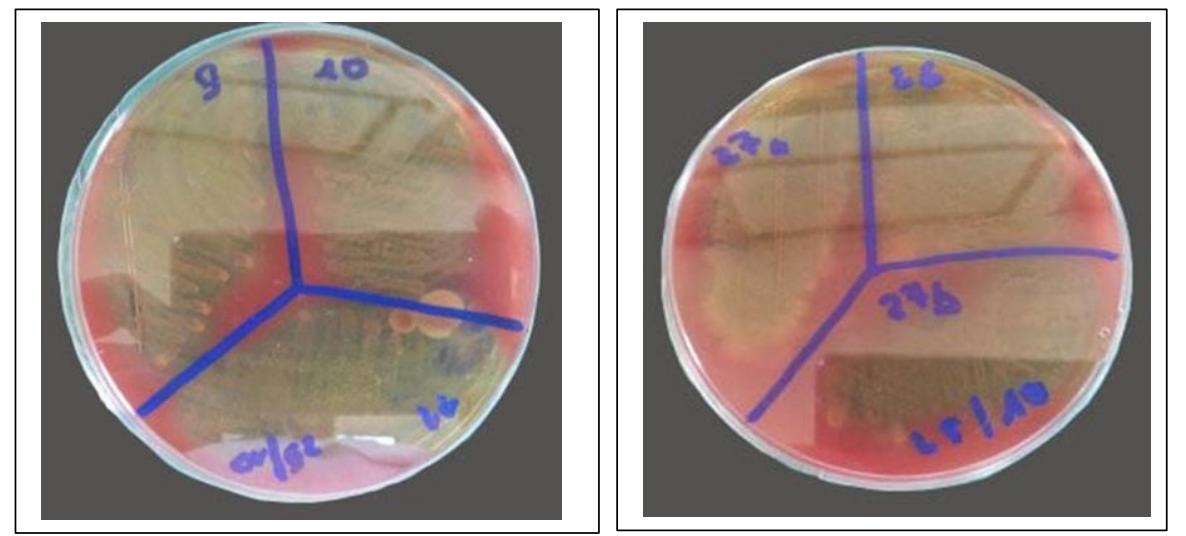

Figure 3: Photographie présentant l'aspect des colonies obtenues sur la gélose au sang après incubation (Cl. Abé, 2016). 
Tableau 1: Pourcentage de résultats (\%) des caractères étudiés de la galerie type Bacillus.

\begin{tabular}{lcccc}
\hline Caractères recherchés & Positif $(\%)$ & Négatif (\%) & variable (\%) & Non déterminé \\
\hline Utilisation du lactose & 0 & $96 \%(50)$ & 0 & $4 \%(2)$ \\
Fermentation du glucose & $79 \%(41)$ & 0 & $17 \%(9)$ & $4 \%(2)$ \\
Production d' $\mathrm{H}_{2} \mathrm{~S}$ & 0 & $96 \%(50)$ & & $4 \%(2)$ \\
Production de gaz & 0 & $96 \%(50)$ & & $4 \%(2)$ \\
Citrate perméase & $69 \%(36)$ & 0 & $27 \%(14)$ & $4 \%(2)$ \\
Lécithinase & $56 \%(29)$ & $40 \%(21)$ & 0 & $4 \%(2)$ \\
Fermentation du mannitol & $13 \%(7)$ & $56 \%(29)$ & $27 \%(14)$ & $4 \%(2)$ \\
Nitrate réductase & $69 \%(36)$ & 0 & $27 \%(14)$ & $4 \%(2)$ \\
Mobilité & $96 \%(50)$ & 0 & 0 & $4 \%(2)$ \\
Uréase & $13 \%(7)$ & $83 \%(43)$ & 0 & $4 \%(2)$ \\
Tryptophanase & 0 & $96 \%(50)$ & 0 & $4 \%(2)$ \\
Coloration de Gram & $100 \%(52)$ & $0 \%$ & & \\
Mobilité & $100 \%(52)$ & $0 \%$ & & $4 \%(2)$ \\
Test de la catalase & $96 \%(50)$ & 0 & & $4 \%(2)$ \\
Test de l'oxydase & $13 \%(7)$ & $23 \%(12)$ & $60 \%(31)$ & $0 \%$ \\
Croissance en milieu & 0 & $13 \%(7)$ & $87 \%(45)$ & \\
anaérobie & & & & \\
\hline
\end{tabular}

$\mathrm{N}=52$ souches de Bacillus

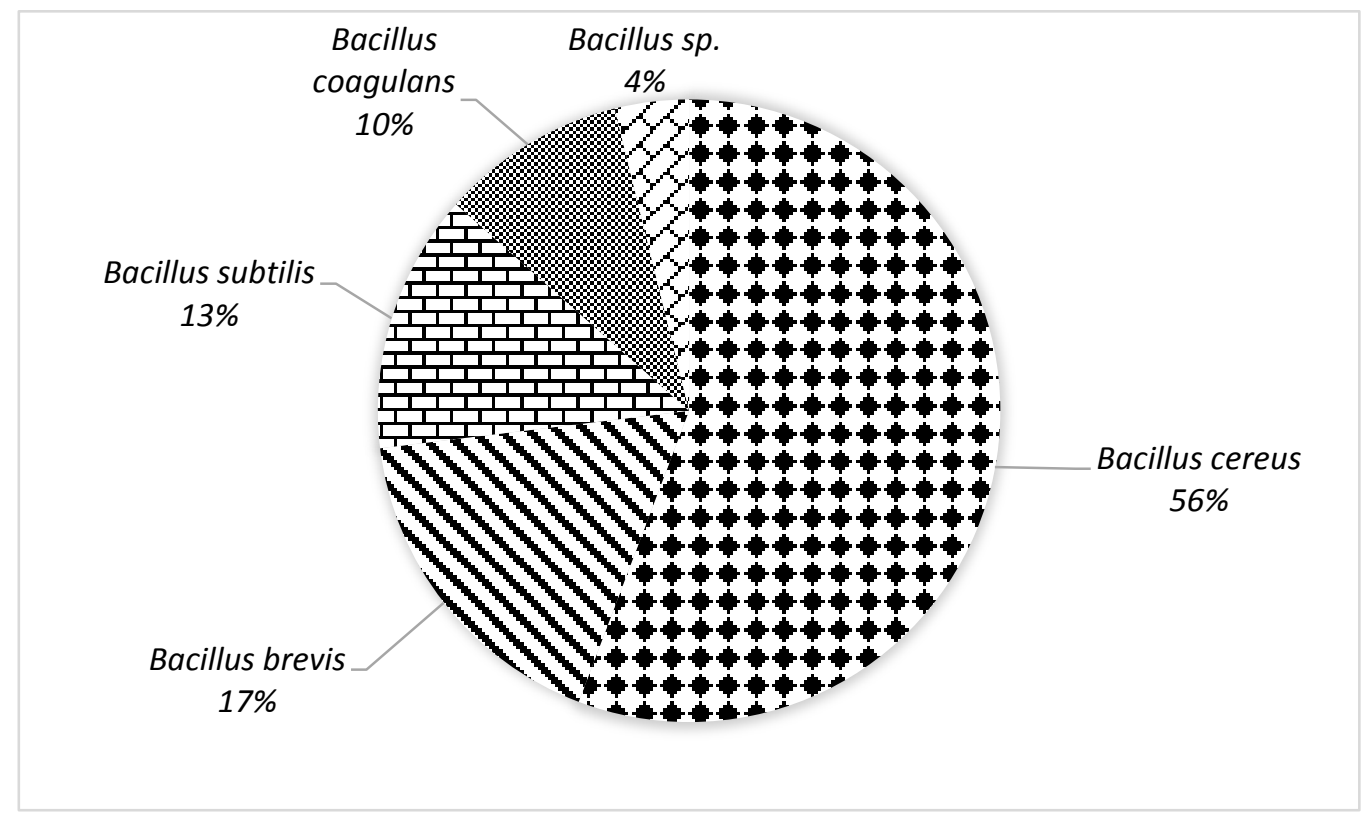

Figure 4: Fréquences relatives des espèces identifiées à partir de 52 souches de Bacillus isolées à partir de racines de manioc en Côte d'Ivoire. 


\section{DISCUSSION}

Les différentes espèces de Bacillus identifiées dans cette étude sont similaires aux travaux de Assanvo et al., 2006 et Coulin et al., 2006 . Le manioc est donc un milieu propice pour la croissance des Bacillus à cause du substrat nutritif qui s'y trouve (Obilie et al., 2003; Assanvo et al., 2006).

Une forte présence de Bacillus cereus dans le manioc est une situation à considérer lorsqu'on sait que ces microorganismes peuvent poser un problème de santé. En effet, ils sont fortement impliqués dans les cas de toxiinfections alimentaires. Notons toutefois que des cas d'intoxication par les bacilles du groupe cereus sont souvent non déclarés, mal diagnostiqués donc sous-évalués en Afrique et autres pays en développement (Kotiranta et al., 2000). Par contre selon Merzougui et al. (2014), beaucoup de cas d'intoxications alimentaires par Bacillus cereus ont été signalés ailleurs dans le monde. Cette bactérie a été la deuxième cause des toxi-infections alimentaires en France en 2012 (InVS, 2012). Sa prévalence dans les aliments à base de végétaux est conférée par les spores (Guinebretière et al., 2002).

En Côte d'Ivoire, jusqu'en 2010, aucun cas d'intoxication associé au groupe B. cereus n'a été déclaré (MSLS, 2010). Cependant, Kouamé et al. (2012) ont isolé des spores de $B$. cereus dans l'attiéké commercial produit dans le Sud de la Côte d'Ivoire. Cette présence devrait interpeller lorsque nous savons que le manioc est l'un des aliments les plus prisés en Côte d'Ivoire et qu'il est consommé frais dans certaines régions du pays notamment à l'Ouest.

En plus du problème de santé que $B$ cereus pourrait poser, cette bactérie peut altérer les caractéristiques organoleptiques des produits alimentaires par la production de lipases et de protéases. Ainsi, si l'on devait commercialiser des produits dérivés du manioc, ce problème devra être pris en compte pour offrir aux consommateurs des produits de qualité, exempt de toutes impuretés qui pourraient mettre en doute la crédibilité sanitaire et organoleptique du produit.
Bacillus brevis, représente environ $18 \%$ des souches identifiées. Cette espèce peut être trouvée dans le sol, l'air, la poussière, le lait et le fromage et produit un antibiotique appelé tyrothricine. La tyrothricine est un mélange polypeptidiqueantibiotique cyclique. Il s'agit d'un antibiotique localement efficace contre les bactéries gram-positives comme Staphylococcus, Clostridium, Listeria. Il est parfois combiné avec la benzocaine pour soulager les maux de gorge.

Bacillus subtilis représenté en minorité dans la matrice alimentaire étudiée, constitue un agent de lutte biologique potentiel contre les maladies fongiques des plantes grâce à la sécrétion extracellulaire d'un certain nombre d'antibiotiques, de lipopeptides microbiens (Deravel et al., 2014) et d'enzymes hydrolytiques tels que les chitinases et les protéases. Les champignons phytopathogènes et les oomycètes présentent un impact négatif sur la sécurité alimentaire.

Le contrôle des maladies des plantes par un contrôle biologique en utilisant les ennemis naturels de ces ravageurs, tels que les rhizobactéries comme les Bacillus serait l'idéal De plus, B. subtilis colonise efficacement la rhizosphère, ce qui lui confère des avantages en tant que biopesticide potentiel et biofertilisant. Par conséquent, ce microorganisme peut favoriser la croissance des plantes en augmentant la disponibilité de l'azote et du phosphore dans les sols agricoles (Rekha et al., 2018). Cela peut contribuer à augmenter les rendements des cultures de manière durable tout en éliminant progressivement l'utilisation de pesticides chimiques.

Bacillus coagulans est une souche probiotique puissante qui est prescrite en thérapeutique aux patients ayant besoin d'un soutien immunitaire aigu (Hun, 2009). Ce probiotique est extrêmement robuste et peut facilement survivre à l'acide gastrique pour coloniser l'ensemble du tractus gastrointestinal. 


\section{Conclusion}

Dans le but de connaître l'importance relative des Bacillus dans le manioc, une étude morphologique et biochimique à partir de 52 souches de Bacillus isolés du manioc frais a permis de mettre en évidence des espèces de Bacillus comme Bacillus cereus, Bacillus brevis, Bacillus subtilis, Bacillus coagulans et Bacillus sp. La prédominance de Bacillus cereus sur ou dans les racines fraîches de manioc consommées crues après récolte constitue une source de danger potentiel pour la santé des consommateurs réguliers des racines de manioc cru, notamment en raison de son implication dans de nombreux cas de TIA.

\section{CONFLIT D'INTERETS}

Les auteurs déclarent qu'ils n'ont aucun conflit d'intérêts.

\section{CONTRIBUTIONS DES AUTEURS}

AMKA et JBA ont élaboré le protocole qui a été validé par JBA, MS et KMK. L'analyse des résultats a été supervisée par JBA et MS et validée par KMK. JBA et AMKA ont participé à la rédaction de l'article. KMK, MS et JBA ont contribué à l'amélioration du manuscrit.

\section{REMERCIEMENTS}

Les auteurs remercient le LANADA (Côte d'Ivoire) pour son appui technique dans la réalisation de ce travail.

\section{REFERENCES}

Aboussaid H, El Messoussi S, Oufdou K. 2009. Activité insecticide d'une souche marocaine de Bacillus thuringiensis sur la mouche méditerranéenne: Ceratitis capitata (Wied.) (Diptera: Tephritidae). Afrique SCIENCE, 05(1): 160-172.

Agre AP, Kouchade S, Odjo T, Dansi M, Nzobadila B, Assogba P, Dansi A, Akoegninou A, Sanni A. 2015. Diversité et évaluation participative des cultivars du manioc (Manihot esculenta Crantz) au Centre Bénin. Int. J. Biol. Chem. Sci.
9(1): $\quad 388-408 . \quad$ DOI: http://dx.doi.org/10.4314/ijbcs.v9i1.33

Amoa-Awua WK, Terlabie NN, SakyiDawson E. 2006. Screening of 42 Bacillus isolates for ability to ferment soybeans into dawadawa. Int. J. Food Microbiol., 106(3): 343-347.

Assanvo JB, Agbo NG, Behi YEN, Coulin P, Farah Z. 2006. Microflora of traditional starter made from cassava for "attiéké" production. Food Control., 17(2006): 37-41. DOI: $10.1016 /$ j.foodcont. 2004.08.006

Azokpota P, Hounhouigan DJ, Nago MC, Jakobsen M. 2006. Esterase and protease activities of Bacillus spp from afitin, iru and sonru, three African locust bean (Parkia biglobosa) condiments from Benin. Afr. J. Biotechnol., 5(3): 265-272. Azokpota P, Moller PL, Hounhouigan JD, Jakobsen M. 2007. Biodiversity of predominant Bacillus isolated from afitin, iru and sonru at different fermentation time. Int. J. Biol. Chem. Sci., 1(3): 211-222.

Azokpota P, Hounhouigan DJ, Annan NT, Nago MC, Jakobsen M. 2008. Diversity of volatile compounds of afitin, iru and sonru, three fermented food condiments from Benin. World. J. Microbiol. Biotechnol., 24(6): 879-885.

Barrow GI, Feltham RK. 1993. Cowan and Steel's Manual for the Identification of Medical Bacteria ( $3^{\text {rd }}$ edn). Cambridge University Press: Cambridge, UK.

Burns AE, Gleadow RM, Zacarias AM, Cuambe CE, Miller RE, Cavagnaro TR. 2012. Variations in the chemical composition of cassava (Manihot esculenta, Crantz) leaves and roots as affected by genotypic and environmental variation. J. Agric. Food Chem., 60: 4946-4956, dx.doi.org/10.1021/ jf2047288 
Coulin P, Farah Z, Assanvo J, Spillmann H, Puhan Z. 2006. Characterisation of microflora of attiéké, a fermented cassava product, during traditional smallscale preparation. Int. J. Food Microbiol., 106 (2006): 131-136. DOI:10.1016/j.ijfoodmicro.2005.06.012.

Deravel J, Lemière S, Coutte F, Krier F, Van Hese N, Béchet M, Sourdeau N, Höfte M, Leprêtre A, Jacques P. 2014. Mycosubtilin and surfactin are efficient, low ecotoxicity molecules for the biocontrol of lettuce downy mildew. Appl. Microbiol. Biotechnol., 98(14): 6255-6264. DOI 10.1007/s00253-0145663-1.

FAO. 2016. FAOSTAT Division de la Statistique. Agriculture Organization of the United Nations FAO. http://faostat 3.fao.org/browse/Q/QC/F.

Hun L. 2009. Bacillus coagulans significantly improved abdominal pain and bloating in patients with IBS. Postgrad Med., 121(2):119-24. DOI: 10.3810/pgm. 2009.03.1984.

Hafidi Z, Handor H, Laghmari M, Handor N, Cherkaoui OL, Tachfouti S, Seffar M, Daoudi R. 2013. Diagnostic et traitement de la Maladie du charbon à localisation palpébrale: à propos d'un cas et revue de littérature. Pan. Afr. Med. J., 15: 1-9. DOI: 10.11604/pamj.2013.15.94.2720

InVS (Institut de Veille Sanitaire). 2012. Surveillance des toxi-infections alimentaires collectives Données de la déclaration obligatoire. InVS. http://invs.santepubliquefrance.fr/Dossie rs-thematiques/Maladies-

infectieuses/Maladies-a-declarationobligatoire/Toxi-infections-alimentairescollectives/Donnees-epidemiologiques

Kakou CA, Kambire O, Boli AZB, Yoro DT, Koffi RN, Koussemon M. 2017. Diversity and enzymatic characterization of Bacillus species isolated from traditional cassava starters used for attiéké production. Int. J. Biol. Chem. Sci., 11(2): 531-540. DOI: https://dx.doi.org/10.4314/ijbcs.v11i2.1

Kouamé AK, Djéni TN, N'Guessan FK, Djè KM. 2012. Postprocessing microflora of commercial attiéké (a fermented cassava product) produced in the south of Côte d'Ivoire. Lett. Appl. Microbiol., 56(1): 44-50. DOI: 10.1111/lam.12014

Kotiranta A, Lounatmaa K, Haapasalo M. 2000. Epidemiology and pathogenesis of B. cereus infections. Microbes Infect., 2(2):189-198. DOI: $\quad 10.1016 / \mathrm{s} 1286$ 4579(00)00269-0

Guinebretiere M, Broussolle HV, Nguyen-The C. 2002. Enterotoxigenic profiles of food-poisoning and food-borne Bacillus cereus strains. J. Clin. Microbiol., 40(8): 3053-3056. DOI: 10.1128/ JCM.40.8.3053-3056.2002

Guinebretière $\mathrm{MH}$, Velge $\mathrm{P}$, Couvert $\mathrm{O}$, Carlin F, Debuyser ML, Nguyen-The C. 2010. Ability of Bacillus cereus group strains to cause food poisoning varies according to phylogenetic affiliation (groups I to VII) rather than species affiliation. J. Clin. Microbiol., 48: 33883391.

Merzougui S, Lkhider M, Grosset N, Gautier M, Cohen N. 2014. Prevalence, PFGE typing and antibiotic resistance of Bacillus cereus group isolated from food in Morocco. Foodborne Pathog. Dis., 11(2): 145-149.

MSLS (Ministère de la santé et de la lutte contre le Sida). 2010. Plan d'Action National de Sécurité Sanitaire des Aliments (PANSSA) couvrant la période quinquennale 2011-2015. Côte d'Ivoire. http://extwprlegs1.fao.org/docs/pdf/ivc1 47264.pdf

Obilie ME, Tano-Debrah K, Amoa-Awua KW. 2003. Microbial modification of the texture of grated cassava during 
fermentation into akyeke. Int. J. Food

Microbiol., $\quad$ 89(2-3): $\quad 275-280$

https://doi.org/10.1016/S0168-

1605(03)00294-0

Oyeleke SB, Ibrahim AD, Manga SB, Rabah

AB, Auta H, Ladan F. 2011. Production

of bacterial amylase by Bacillus species isolated from rice husk dumpsites in Sokoto metropolis, Nigeria. Int. J. Biol. Chem. Sci., 5(1): 380-385.

Parkouda C, Nielsen DS, Azokpota P, Ouoba LII, Amoa-Awua WK, Thorsen L, Hounhouigan JD, Jensen JS, TanoDebrah K, Diawara B, Jakobsen M. 2009. The microbiology of alkalinefermentation of indigenous seeds used as food condiments in Africa and Asia. Critical Reviews Microbiol., 35(2): 139156. DOI: $10.1080 / 10408410902793056$

PISTRETTO. 2007. Infos pour les apprenants: Manioc - Récolte abondante des Tropiques.

PISTRETTO. http://www.pistor.ch/lebensmittel/PDF/A liments/Manioc.pdf

Rekha K, Baskar B, Srinath S, Usha B. 2018. Plant-growth-promoting rhizobacteria Bacillus subtilis RR4 isolated from rice rhizosphere induces malic acid biosynthesis in rice roots. Can. J.
Microbiol., $\quad$ 64(1):20-27. DOI: 10.1139/cjm-2017-0409.

Stein T. 2005. Bacillus subtilis antibiotics: structures, syntheses and specific functions. Molecular Microbiology, 56(4): $845-857$. DOI:10.1111/j.13652958.2005.04587.x

Vinoj G, Vaseeharan B, Jayaseelan DB, Rajakumaran P, Ravi C. 2013. Inhibitory effects of Bacillus licheniformis (DAB1) and Pseudomonas aeruginosa (DAP1) against Vibrio parahemolyticus isolated from Fenneropenaeus indicus. Aquacult. Int., 21(5):1121-1135. https://doi.org/ 10.1007/s10499-012-9617-2

Waites MJ, Morgan NL, Rockey JS, Higton G. 2001. Industrial Microbiology: An Introduction. Blackwell science Ltd: Oxford, UK.

Yobouet A, Dadie A, Traore S, Dje K, Bonfoh B. 2016. Contamination par Bacillus cereus de l'attiéké produit dans le secteur informel au sud de la Côte d'Ivoire et gestion du risque par le réchauffage hydrothermique. International Journal of Innovation and Applied Studies, 15(3): 637-654. 